\title{
Entangling power of permutation-invariant quantum states
}

\author{
Vladislav Popkov* \\ Institut für Theoretische Physik, Universität zu Köln, Zülpicher Strasse 77, 50937 Köln, Germany
}

Mario Salerno ${ }^{\dagger}$

Dipartimento di Fisica “E.R. Caianiello,” and Istituto Nazionale di Fisica Nucleare (INFN) Sezione di Napoli-Gruppo Collegato di Salerno, Consorzio Nazionale Interuniversitario per le Scienze Fisiche della Materia (CNISM), Universitá di Salerno, I-84081 Baronissi (SA), Italy

\author{
Gunter Schütz \\ Institut für Festkörperforschung, Forschungszentrum Jülich, 52425 Jülich, Germany
}

(Received 18 April 2005; published 22 September 2005)

\begin{abstract}
We investigate the von Neumann entanglement entropy as function of the size of a subsystem for permutation invariant ground states in models with finite number of states per site, e.g., in quantum spin models. We demonstrate that the entanglement entropy of $n$ sites in a system of length $L$ generically grows as $\sigma \log _{2}[2 \pi e n(L-n) / L]+C$, where $\sigma$ is the on-site spin and $C$ is a function depending only on magnetization.

DOI: 10.1103/PhysRevA.72.032327

PACS number(s): 03.67.Hk, 03.65.Ud, 75.10.Jm
\end{abstract}

\section{INTRODUCTION}

Recently it has been argued that for critical (gapless) quantum spin systems the entanglement entropy of a block of $n$ spins diverges logarithmically as $\gamma \log _{2} n$, while for noncritical systems it converges to a constant finite value [1-3]. This property was interpreted in the framework of conformal field theory [4] associated with the corresponding quantum phase transition and the prefactor $\gamma$ of the logarithm was related to the central charge $c$ of the theory as $c=3 \gamma$. This was shown explicitly for the exactly solvable antiferromagnetic Heisenberg spin 1/2 chain, i.e., the $X X Z$ model where the anisotropy parameter $\Delta$ belongs in the critical regime to the interval $(-1,1)$ and $\gamma=1 / 3$. Rather surprisingly, at the transition point $\Delta=-1$ from gapless to noncritical behavior, the entanglement (von Neumann) entropy of a block of spins in the ground state was found to grow faster than in the critical domain $-1<\Delta \leqslant 1$, namely as $\gamma \log _{2} n$ with the logarithmic prefactor satisfying $\frac{1}{2} \leqslant \gamma \leqslant 1$ [5]. At $\Delta=-1$ the ground state of the $X X Z$ Hamiltonian has permutational invariance (up to a gauge transformation), and is degenerate with respect to the total $z$ magnetization, so that the whole system is generically described via a density matrix which can be written as a (weighted) sum of projectors on the multiplet components. The lower bound $\gamma=\frac{1}{2}$ is attained if the state of the whole system is a pure state (only one component of the multiplet is present), while the upper bound $\gamma=1$ is reached for a mixed state where all the components of the multiplet have equal weights. Note that the pure state is generically not factorizable, see Eq. (4) below, thus producing a mixed state after partial tracing.

In the present paper we generalize the approach of [5] to arbitrary permutation-invariant quantum spin states. In particular, we consider the case of a ferromagnetic spin chain

\footnotetext{
*Email address: popkov@thp.uni-koeln.de
}

with arbitrary spin $\sigma$ on every site and show that the entanglement entropy of a block of $n$ spins in the ferromagnetic ground state generically diverges as $\sigma \log _{2} n$. Our approach uses the invariance of the ground state under site permutations, thus allowing us to compute the entanglement entropy exactly for blocks of arbitrary size and for systems of arbitrary length.

The paper is organized as follows. In Sec. II we introduce the permutation invariant states and list physical systems whose ground states have this symmetry. In Sec. III we formulate a theorem which gives the analytical expression of the eigenvalues of the reduced density matrix for arbitrary spin $\sigma$. Using this theorem we compute the entanglement entropy of a block of size $n$ in the finite system of total length $L$ in specific ground state sectors. Taking the limit of large subsystem sizes, we derive analytic expressions for the entanglement entropy $S_{(n)}$ both for $n, L \gg 1$ and for $n \gg 1$, $L=\infty$. As a result, we find that in the ground state sector with a fixed value of $S^{z}$ the block entanglement entropy diverges for large $n$ as $S_{(n)}=\sigma \log _{2}[2 \pi e n(L-n) / L]+C$. In Sec. IV the case of spin $\sigma \geqslant 1$ is treated in more detail. A discussion and some further remarks close the paper.

\section{PERMUTATION INVARIANT STATES}

Let us consider states in a Hilbert space $\left(\mathrm{C}^{d}\right)^{\otimes L}$ of a quantum spin chain of local spin $\sigma$, where $d=2 \sigma+1$ is the dimension of the spin space at every site $i$ and $d^{L}$ the dimension of the whole Hilbert space. Permutation-invariant states constitute a subspace $Q$ of substantially smaller dimension

$$
\kappa(L)=\operatorname{dim} Q=\left(\begin{array}{c}
L+2 \sigma \\
2 \sigma
\end{array}\right),
$$

this being the number of possible ways to distribute $L$ indistinguishable objects among $2 \sigma+1$ boxes. Denoting by $N_{j}$ the number of objects in the block $j$, the state of the whole system is completely characterized by 


$$
\begin{aligned}
&\left|\Psi\left(L, N_{0}, N_{1}, \ldots, N_{2 \sigma}\right)\right\rangle \\
&= \sqrt{\frac{N_{0} ! N_{1} ! \ldots N_{2 \sigma} !}{L !}} \\
& \times \sum_{P} \mid \underbrace{\downarrow \downarrow \ldots \downarrow}_{N_{0}} \ldots \underbrace{\nearrow \nearrow \ldots \nearrow}_{N_{i}} \ldots \underbrace{\uparrow \ldots \uparrow}_{N_{2 \sigma}},
\end{aligned}
$$

where $N_{0}$ is the number of spins pointing down $\left(\sigma_{z}=-\sigma\right), N_{j}$ is the number of spins with $\sigma_{z}=-\sigma+j$, up to $N_{2 \sigma}$ spins with maximal $\sigma_{z}=\sigma$, occupying in total $L=N_{0}+N_{1}+\ldots+N_{2 \sigma}$ sites. The sum is taken over all possible permutations, the total number of which is $L ! / N_{0} ! N_{1} ! \ldots N_{2 \sigma} !$, with the prefactor in Eq. (2) taking care of the normalization.

We will be interested in the entanglement of a block of $n$ spins with the remaining $L-n$ spins (playing here the role of the environment), characterized by the von Neumann entropy

$$
S_{(n)}=-\operatorname{tr}\left(\rho_{n} \log _{2} \rho_{n}\right)=-\sum_{k} \lambda_{k} \log _{2} \lambda_{k},
$$

where $\rho_{n}$ is the reduced density matrix of the block, obtained from the density matrix $\rho$ of the whole system by tracing out the degrees of freedom of the environment $\rho_{(n)}=\operatorname{tr}_{(L-n)} \rho$, and $\lambda_{k}$ are its eigenvalues. The density matrix of the whole system is a projector on the pure state in Eq. (2), i.e., $\rho=\left|\Psi\left(L, N_{0}, N_{1}, \ldots N_{2 \sigma}\right)\right\rangle\left\langle\Psi\left(L, N_{0}, N_{1}, \ldots N_{2 \sigma}\right)\right|$. Notice that due to the permutational symmetry, $S_{(n)}$ does not depend on the particular choice of the sites in the block but only on its size $n$. The eigenvalues $\lambda_{k}$ of the reduced density matrix are by construction all real, non-negative, and sum up to one: $\sum_{k} \lambda_{k}=1$.

Before giving the general expression for $\lambda_{k}$ we discuss separately the two-states case of $\sigma=1 / 2$. In this case the state of the system Eq. (2) reduces to

$$
|\Psi(L, N)\rangle=\left(\begin{array}{c}
L \\
N
\end{array}\right)^{-1 / 2} \sum_{P}|\underbrace{\uparrow \uparrow \ldots \uparrow}_{N} \underbrace{\downarrow \downarrow \ldots \downarrow}_{L-N}\rangle,
$$

where $\uparrow$ and $\downarrow$ denote spin up and spin down, respectively. This state appears in the literature in different physical situations. Since the entanglement properties do not depend on the underlying model but only on the form of the wave function, we list some models for which Eq. (4) is an exact ground state to show the diversity of applications.

(i) The isotropic Heisenberg ferromagnet, , where $\sigma_{i}$ are Pauli matrices, $J<0$ denotes the exchange constant, and $L$ is the total number of spins. (We assume periodic boundary conditions $L+1 \equiv 1$.) The ground state belongs to a multiplet of total spin $S=L / 2$ and is $(L+1)$-fold degenerate with $S^{z}=-L / 2,-L / 2+1, \ldots L / 2$. The state Eq. (4) is a pure state, corresponding to the multiplet component with a fixed number $N$ of spins up.

(ii) The Heisenberg antiferromagnet at $\Delta=-1$. In this case, the state (4) is obtained from the ground state by a unitary transformation, inverting every other spin along the chain (note that the von Neumann entropy is invariant under unitary transformations of the state of the system). (iii) The generalized Hubbard model in the limit of strong attraction (the so-called eta-pairing states [6]).

(iv) Hardcore bosons on a complete graph [7-9].

(v) The Lipkin-Meshkov-Glick model [11].

The state (4) also appears in a classical context as stationary distribution of the asymmetric exclusion process (ASEP) describing a Markov process of nonequilibrium stochastic motion of $N$ particles on a ring with $L$ sites with hardcore exclusion. In this case Eq. (4) means that all particle configurations have equal probabilities in the stationary state [10]. The block entanglement entropy for the case of spin $\sigma=1 / 2$ was obtained in [5]. In particular, it was shown that the eigenvalues $\lambda_{k}$ of the reduced density matrix $\rho_{n}$ are $\lambda_{k}(L, n)=C_{k}^{n} C_{N-k}^{L-n} / C_{N}^{L}$, where $C_{m}^{n}$ denotes the binomial coefficient $n ! /[m !(n-m) !]$ and $k=0,1, \ldots \min (n, N)$. In the limit of large $n$, the von Neumann entropy was found to be

$$
S_{(n)}(p) \approx \frac{1}{2} \log _{2}(p q)+\frac{1}{2} \log _{2} 2 \pi e \frac{n(L-n)}{L},
$$

where $p=N / L, q=1-p$. In the next section we generalize these results to the case of arbitrary spins.

\section{ENTANGLEMENT ENTROPY FOR ARBITRARY ON-SITE SPIN $\sigma$}

We formulate the main result of this section as follows:

Theorem. The eigenvalues of the reduced density matrix $\rho_{n}\left(N_{0}, N_{1}, \ldots N_{2 \sigma}\right)$ of a block of $n$ spins in the permutation invariant state (2) of the whole system are given by

$$
\lambda_{\mathbf{k}}(L, n, \sigma)=\left(\begin{array}{c}
L \\
n
\end{array}\right) \prod_{i=0}^{2 \sigma}\left(\begin{array}{c}
N_{i} \\
k_{i}
\end{array}\right)
$$

where $k_{i}, N_{i}$ satisfy $k_{0}+\ldots+k_{2 \sigma}=n, N_{0}+\ldots+N_{2 \sigma}=L$. Here and below we denote the set of $k_{i}$ by the bold $\mathbf{k}$. To prove the theorem we note that the reduced density matrix $\rho_{n}$ is decomposed with respect to symmetric orthogonal subspaces of the system of $n$ spins, classified by the numbers $k_{j}=0,1, \ldots \min \left(n, N_{j}\right)$ of spins with $\sigma_{z}=-\sigma+j$ in the block

$$
\rho_{n}(N)=\sum_{\mathbf{k}} w_{\mathbf{k}}|\psi(n, \mathbf{k})\rangle\langle\psi(n, \mathbf{k})| .
$$

Here the state $|\psi(n, \mathbf{k})\rangle$ has the same structure as Eq. (2)

$$
|\psi(n, \mathbf{k})\rangle=\sum_{P} \mid \underbrace{\downarrow \downarrow \ldots \downarrow}_{k_{0}} \cdots \underbrace{\nearrow \nearrow \ldots \nearrow}_{k_{i}} \ldots \underbrace{\uparrow \uparrow \ldots \uparrow\rangle}_{k_{2 \sigma}}
$$

and $w_{k}$ is the corresponding probability, given by the number of ways one can distribute $N_{j}$ spins (of values $-\sigma+j$ ) on $k_{j}$ sites for all possible values of $N_{j}, k_{j}, j=0, \ldots, 2 \sigma$, divided by the number of ways one can distribute $N=N_{0}+\ldots+N_{2 \sigma}$ spins on $n=k_{0}+\ldots+k_{2 \sigma}$ sites (the total number of states), i.e.,

$$
w_{\mathbf{k}}=\left(\begin{array}{l}
L \\
n
\end{array}\right) \prod_{j=0}^{2 \sigma}\left(\begin{array}{c}
N_{j} \\
k_{j}
\end{array}\right) .
$$

Since the states $|\psi(n, \mathbf{k})\rangle$ are orthogonal, the representation in Eq. (7) is diagonal and the $w_{\mathbf{k}}$ coincide with the eigenvalues of $\rho_{n}(N)$. This proves of the theorem. Notice that for the 


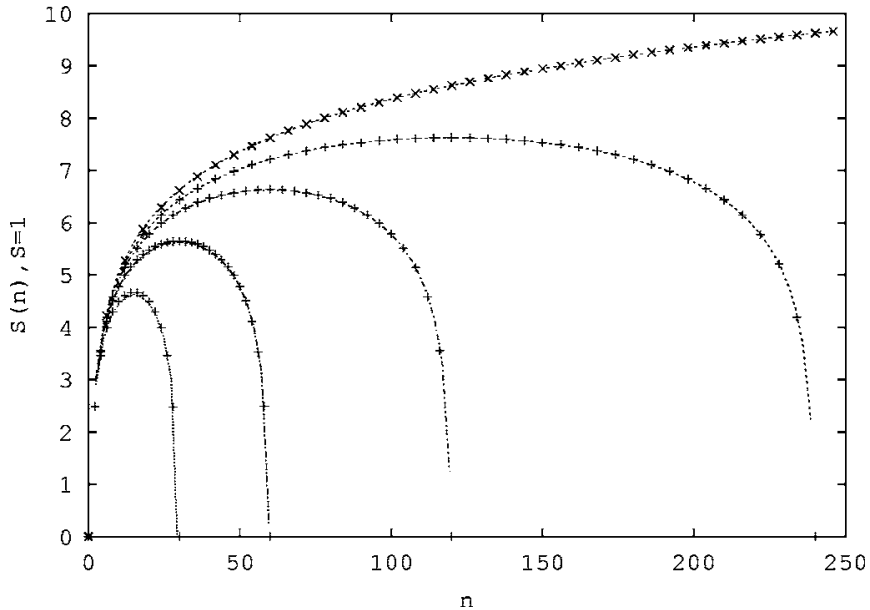

FIG. 1. Exact (points) vs analytical (curves) comparison of von Neumann entropy $S_{(n)}$ for spin $\sigma=1$ in systems with $L=30,60,120,240, \infty$. Blocks of all sizes $0 \leqslant n \leqslant L$ (for finite $L$ ) are considered. The other parameters $N_{1} / L=N_{2} / L=\frac{1}{3}$.

case $\sigma=1 / 2 \mathrm{Eq}$. (6) reproduces the results derived in [5] (use $N_{0}=L-N_{1}, k_{0}=n-k_{1}$ and the invariance under the exchange of $N_{1}$ and $n$ ). Having found the eigenvalues of $\rho_{n}(N)$ one can compute the entanglement entropy $S_{(n)}$ for arbitrary $L, n$, and $N$. In the large- $n$ limit one concludes (see the Appendix) in analogy with the case of $\sigma=1 / 2$ [5], that

$$
\begin{gathered}
S_{(n)}(L, \sigma)=C+\sigma \log _{2}\left(2 \pi e \frac{n(L-n)}{L}\right), \quad n \gg 1, \\
C=\frac{1}{2} \log _{2}\left(\prod_{k=0}^{2 \sigma} p_{k}\right),
\end{gathered}
$$

where $p_{k}=N_{k} / L$. In Figs. 1 and 2 we compare the exact entropy of finite systems, as computed from the exact expres-

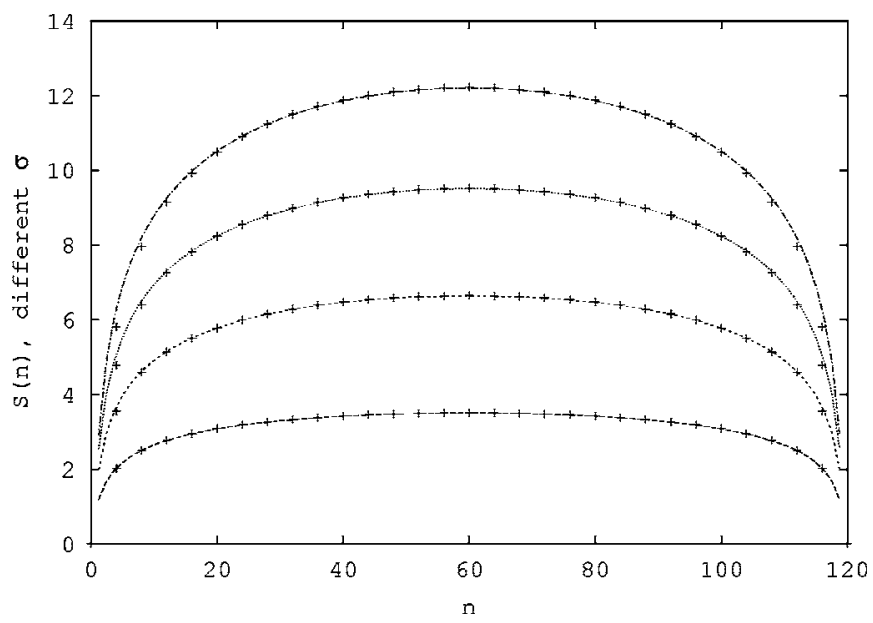

FIG. 2. Entanglement entropy as function of a number of sites $n$ in the block, for different on-site spin $\sigma=\frac{1}{2}, 1, \frac{3}{2}, 2$, for finite system $L=120$. Comparison of the exact formula (points) with the limiting expression (10) (continuous curves). In all cases equal partitions of particles is taken $p_{i}=N_{i} / L=1 /(2 \sigma+1)$. sions Eqs. (3) and (6), with the analytical expression (10), from which we see that there is an excellent agreement also for small values of $n \prod_{k=0}^{2 \sigma} p_{k}$. In the thermodynamic limit $L \rightarrow \infty, N_{i} / L \rightarrow p_{i}$, the eigenvalues of the reduced density matrix (6) simplify to

$$
\lambda_{\mathbf{k}}\left(\infty, n,\left\{p_{i}\right\}_{i=0}^{2 \sigma}\right)=n ! \prod_{i=0}^{2 \sigma} \frac{\left(p_{i}\right)^{k_{i}}}{k_{i} !} .
$$

The entanglement entropy then becomes

$$
S_{(n)}\left(\infty,\left\{p_{i}\right\}_{i=0}^{2 \sigma}\right)=C\left(\left\{p_{i}\right\}\right)+\sigma \log _{2}(2 \pi e n),
$$

where the constant $C$ is given by Eq. (11).

Notice that while the general formulae Eqs. (3) and (6) are valid for an arbitrary choice of parameters, the analytical result (10) is valid for $n \Pi_{k=0}^{2 \sigma} p_{k} \gg 1$. If in the limit of an infinite system $L \rightarrow \infty$ some of the $p_{k}$ vanish (say, $z$ coefficients $p_{k} \rightarrow 0$ out of a total number of coefficients $2 \sigma$ ), the entangled state of $n$ spins will effectively behave like the one with effective site spin $\widetilde{\sigma}=\sigma-z / 2$, and the von Neumann entropy will, respectively, grow as $S_{n} \sim(\sigma-z / 2) \log _{2} n$ instead of $S_{n} \sim \sigma \log _{2} n$ [see Eq. (12)].

It is instructive to recall that Eq. (10) is derived under the assumption of a pure state of the global system. If the state of the whole system is mixed in the ensemble of states with the same symmetry (2), the resulting von Neumann entropy $S_{(n)}$ will become larger. An upper bound of $S_{(n)}$ can be derived by noting that the number of nonzero eigenvalues of the reduced density matrix is equal to the number of terms in the decomposition Eq. (7), which is $\kappa(n)$ [see Eq. (1)]. The upper limit of the entanglement entropy is thus reached when all $\lambda_{k}$ in (3) are equal, implying

$$
\sup \left(S_{(n)}\right)=\log _{2}\left(\begin{array}{c}
n+2 \sigma \\
2 \sigma
\end{array}\right) .
$$

This limit is attained for a mixed state of the global system with all components of the ensemble Eq. (2) equally weighted. In this case the von Neumann entropy is given by the above expression even for finite $n, L$ (see [5] for an example). For large $n, \sup \left(S_{(n)}\right) \approx 2 \sigma \log _{2} n$.

We also remark that in the critical spin models where spin-spin correlation decay algebraically in the ground state (e.g., the region $-1<\Delta \leqslant 1$ of the antiferromagnetic Heisenberg chain), there are three distinct physical properties contributing to the entanglement: (a) on-site correlations due to single-site quantum fluctuations; (b) algebraically decaying spin-spin correlations which survive in the thermodynamic limit; and (c) the correlations due to the constraint of fixed magnetization which vanish in every domain of finite size in the thermodynamic limit. For permutation invariant states (2) only contribution (a) is left in the thermodynamic limit for finite domains, but for domains of finite volume fraction the correlations (c) due to the constraint of fixed magnetization also remain relevant.

\section{MODELS WITH HIGHER SPIN}

As remarked above the entanglement entropy of a quantum state does not depend on any underlying model but only 
on the properties of that state. Nevertheless it is of interest to have some insight for which systems the permutation invariant states (2) considered here are the ground state of that quantum system. First of all, the generalizations of the Sutherland model [13] describing quantum spin chains with an interaction given by the permutation operator in $\mathrm{SU}(N)$,

$$
H=\sum J_{i j} P_{i, j},
$$

are obviously invariant under the permutation group $S_{N}$. Here the set of $J_{k i}$ is defined on any connected graph, an example being nearest neighbor interaction. For ferromagnetic interaction, all $J_{i j}<0$, the states (2) span its ground state, which can be proved along the lines of [14]. For SU(2) and $J_{i j}=J \delta_{i, j+1}$ the Hamiltonian (14) reduces to the isotropic Heisenberg Hamiltonian. For the general case we recall that the permutation operator is written in terms of spin operators as

$$
P=\sum_{i=0}^{2 S}(-1)^{2 S+i} \prod_{k \neq i}^{2 S} \frac{2(\widetilde{\mathbf{S}} \otimes \widetilde{\mathbf{S}})-k(k+1)+2 S(S+1)}{i(i+1)-k(k+1)}
$$

where $N=2 S+1$, (see, e.g., [15]). For the above example, all states (2) are eigenstates of Eq. (14) with the lowest energy.

The increase of entanglement entropy compared to the $s=1 / 2$ case appears to result from the larger local state space of the $\mathrm{SU}(N)$ chain, but not from the $\mathrm{SU}(N)$-symmetry itself. This picture is supported by the generalized disordered $\mathrm{SU}(2)$-symmetric spin- $\sigma$ Heisenberg ferromagnet

$$
H=\sum_{i, j} J_{i, j} g\left(\overrightarrow{\sigma_{i}} \cdot \overrightarrow{\sigma_{j}}-1\right)
$$

where the exchange energies $J_{i, j} \leqslant 0$ may be non-zero between any pair of lattice sites on an arbitrary lattice and $\vec{\sigma}_{i}$ are local SU(2) generators in the spin- $\sigma$ representation. For any polynomial function $g$ with positive expansion coefficients linear combinations of the permutation invariant states (2) with fixed total magnetization are ground states of the Hamiltonian, see [16] for a detailed discussion of the ground states of this model in a probabilistic setting. Since these are not pure states in the sense discussed above (with all quantum numbers $N_{k}$ fixed) the entanglement entropy of these ground states is higher than those of the pure ground states of the $\mathrm{SU}(N)$ spin chain.

Other models with pair interaction, but no symmetry: Using the Perron-Frobenius theorem it is straightforward to construct quantum Hamiltonians of the structure

$$
H=\sum_{i, j} J_{i, j} g_{i, j},
$$

where $g_{i, j}$ is a Hermitian pair interaction matrix satisfying $g_{i, j}|s\rangle=0$ for all $i, j$ and where the wave function $|s\rangle$, which is constant for all spin configurations in $\left(C^{d}\right)^{\otimes L}$, is the ground state of $g_{i, j}$. Then, if all $J_{i, j} \leqslant 0$, the vector $|s\rangle$ is also the ground state of $H$, and if furthermore $g_{i, j}$ has no invariant subspaces, it is the unique ground state with maximal entanglement entropy (13). Such quantum systems also have a probabilistic interpretation as generator of some irreducible Markov chain [10].

\section{SUMMARY AND CONCLUSIONS}

We have obtained exact eigenvalues of the reduced density matrix for permutation invariant states, for arbitrary length of the system. In the thermodynamic limit, it was shown that the von Neumann entropy of entanglement of a block of $n$ spins $\sigma$ with the environment grows logarithmically fast $S_{(n)} \sim \gamma \log _{2} n$, with a prefactor $\gamma=\sigma$ for a pure global state and with $\gamma=2 \sigma$ for homogenously (maximally) mixed global state. Various models, the ground states of which are permutation invariant, are given, for spin $\sigma=1 / 2$ and higher. We note that the logarithmic growth of entanglement entropy due to permutation invariance is faster than the one of critical (conformally invariant) models, where $S_{(n)} \sim \gamma \log _{2} n$ with $\gamma=1 / 3$ was observed [2].

It is also interesting to compare the finite size corrections of the entropy of the permutation-invariant states (10) with those of critical spin chains, $S_{(n)}^{c r} \sim c / 3 \log _{2}[L / \pi \sin (\pi n / L)]$, obtained in [12] [see Eq. (3.8) in this paper]. Expanding in the first nonvanishing order of $1 / L$ in both expressions, we get the finite size corrections $\Delta^{c r}(n)=S_{(n)}^{c r}(L)-S_{(n)}^{c r}(\infty)$, $\Delta^{p e r}(n)=S_{(n)}^{p e r}(L)-S_{(n)}^{p e r}(\infty)$ for permutation invariant and critical models as

$$
\begin{gathered}
\Delta^{p e r}(n)=\sigma \log _{2}\left(1-\frac{n}{L}\right) \approx-\sigma \frac{1}{\ln 2} \frac{n}{L}+O\left(\frac{n}{L}\right)^{2}, \\
\Delta^{c r}(n) \approx \frac{c}{3} \log _{2}\left[1-\frac{1}{3}\left(\frac{\pi n}{L}\right)^{2}\right] \approx-\frac{c}{9} \frac{1}{\ln 2}\left(\frac{\pi n}{L}\right)^{2}+O\left(\frac{n}{L}\right)^{4} .
\end{gathered}
$$

Thus, besides the difference of the coefficient of the principal logarithmic divergence $\left(\gamma \log _{2} n\right.$ with $\gamma=1 / 3$ for critical $X X Z$ and $\gamma=\sigma$ for permutation invariant states, respectively, a substantial difference in the finite size corrections (linear in $n$ and of order $1 / L$ for permutation invariance) and quadratic in $n$ and of order $1 / L^{2}$, respectively (for conformal invariant critical states) is also observed.

\section{ACKNOWLEDGMENTS}

V.P. acknowledges the INFM, Unitá di Salerno, for providing a 3 months grant during which this work was initiated, and the Department of Physics of the University of Salerno for the hospitality. M.S. acknowledges partial financial support from Forschungszentrum Jülich, from the University of Cologne within Grant No. SFB/TR12 "Symmetries and Universality in Mesoscopic Systems" and from Grant No. MURST-PRIN-2003 initiative.

\section{APPENDIX}

To prove Eq. (10) we first give the general scheme and then demonstrate the result on a particular example. For simplicity we shall discuss the limit when the size of the global system tends to infinity, $L \rightarrow \infty$. The eigenvalues (6) $\lambda_{\mathbf{k}}$ $\equiv \lambda\left(k_{0}, k_{1}, \ldots k_{2 \sigma}\right)$ for large values of $n$ and $k_{i}$, can be approximated by a multidimensional Gaussian distribution with mean and moments given by 


$$
\begin{gathered}
\left\langle k_{i}\right\rangle=n p_{i}, \\
\left\langle\left(k_{i}-\left\langle k_{i}\right\rangle\right)^{2}\right\rangle=n p_{i}\left(1-p_{i}\right), \\
\left\langle\left(k_{i}-\left\langle k_{i}\right\rangle\right)\left(k_{j}-\left\langle k_{j}\right\rangle\right)\right\rangle=-n p_{i} p_{j}, \quad i \neq j,
\end{gathered}
$$

where $p_{i}=N_{i} / L, i=0,1, \ldots 2 \sigma$, is the average density of spins $-\sigma+i$ in the system. To prove Eqs. (A1)-(A3) we first observe that, using the Stirling approximation $m !=m^{m} \exp (-m) \sqrt{2 \pi m}$, in the limit $L, N_{i} \rightarrow \infty$ the eigenvalues (6) of the reduced density matrix can be approximated as

$$
\lambda_{\mathbf{k}} \approx c_{\mathbf{k}}(n) \prod_{i=0}^{2 \sigma} p_{i}^{k_{i}}
$$

with $c_{\mathbf{k}}(n)=n ! /\left(k_{0} ! k_{1} ! \ldots k_{2 \sigma} !\right)$. Notice that in this limit the distribution of the $\lambda_{\mathbf{k}}$ coincides with the multinomial distribution

$$
\left(\sum_{i=0}^{2 \sigma} p_{i}\right)^{n}=\sum_{\mathbf{k}} c_{\mathbf{k}}(n) \prod_{i=0}^{2 \sigma} p_{i}^{k_{i}}=\sum_{\mathbf{k}} \lambda_{\mathbf{k}}=1
$$

Using this expression, the mean value of $k_{\alpha}$, $\alpha=0,1, \ldots, 2 \sigma$, can be found as

$$
\left\langle k_{\alpha}\right\rangle=\sum_{\mathbf{k}} k_{\alpha} c_{\mathbf{k}}(n) \prod_{i=0}^{2 \sigma} p_{i}^{k_{i}}=p_{\alpha} \frac{\partial}{\partial p_{\alpha}}\left(\sum_{i=0}^{2 \sigma} p_{i}\right)^{n}=n p_{\alpha} .
$$

In a similar manner we obtain

$$
\begin{gathered}
\left\langle k_{\alpha}\left(k_{\alpha}-1\right)\right\rangle=p_{\alpha}^{2} \frac{\partial^{2}}{\partial p_{\alpha}^{2}}\left(\sum_{i=0}^{2 \sigma} p_{i}\right)^{n}=n(n-1) p_{\alpha}^{2}, \\
\left\langle k_{\alpha} k_{\beta}\right\rangle=p_{\alpha} p_{\beta} \frac{\partial^{2}}{\partial p_{\alpha} \partial p_{\beta}}\left(\sum_{i=0}^{2 \sigma} p_{i}\right)^{n}=n(n-1) p_{\alpha} p_{\beta},
\end{gathered}
$$

from which Eqs. (A2) and (A3) readily follow. Denoting by $x_{i}=k_{i} / n$, so that $0<x_{i}<1$, the eigenvalues $\lambda_{\mathbf{k}}$ are approximated as

$$
\lambda_{\mathbf{k}} \approx \frac{\sqrt{\operatorname{det} A}}{(2 \pi)^{\sigma}} \exp \left(-\frac{1}{2} \sum_{i, j=1}^{2 \sigma} A_{i j}\left(x_{i}-p_{i}\right)\left(x_{j}-p_{j}\right)\right),
$$

where the shifting of $x_{i}, x_{j}$ is introduced to account for the nonzero mean Eq. (A1), and the coefficients of the symmetric matrix $a_{i j}=a_{j i}$ are fixed using the moments Eqs. (A2) and
(A3). On the other hand, the computation of the moments from the distribution Eq. (A5) gives

$$
\left\langle\left(x_{i}-p_{i}\right)\left(x_{j}-p_{j}\right)\right\rangle=\frac{M_{i j}}{\operatorname{det} A},
$$

where $M_{i j}$ are the minors of the matrix $A$. Comparing Eq. (A6) with Eqs. (A2) and (A3), we can fix the elements $A_{i j}$ of the matrix. To illustrate the method let us consider the case of spin $\sigma=1$. Then,

$$
\lambda_{\mathbf{k}} \equiv \lambda(x, y) \approx \frac{\sqrt{D}}{2 \pi} \exp \left(-\frac{1}{2}\left(a x^{2}+2 b x y+c y^{2}\right)\right)
$$

where we introduced $x=x_{1}-p_{1}, \quad y=x_{2}-p_{2}$ and $D=\operatorname{det} A$ $=a c-b^{2}$ for brevity of notation. From Eqs. (A2) and (A3) we have $\left\langle x^{2}\right\rangle=n p_{1}\left(1-p_{1}\right),\left\langle y^{2}\right\rangle=n p_{2}\left(1-p_{2}\right)$, and $\langle x y\rangle=-n p_{1} p_{2}$. On the other hand, from Eq. (A6) we obtain $\left\langle x^{2}\right\rangle=c / D$, $\left\langle y^{2}\right\rangle=a / D$, and $\langle x y\rangle=-b / D$. Computing the determinant

$$
D=a c-b^{2}=D^{2}\left(\left\langle x^{2}\right\rangle\left\langle y^{2}\right\rangle-\langle x y\rangle^{2}\right)
$$

and substituting the moments, we get

$$
D^{-1}=n^{2} p_{1} p_{2} p_{3} \text {. }
$$

The von Neumann entropy is then computed as

$$
S \approx-\int d x \int d y\left[\lambda(x, y) \log _{2} \lambda(x, y)\right] .
$$

Notice that for finite $p_{i}$ the larger contribution to the integral comes from the neighbor of the origin $x=y=0$ so that we can extend the limits of integration to the whole real axis and perform the integral exactly. This leads to

$$
S(n) \approx \log _{2} \frac{2 n \pi e}{\sqrt{D}}=\log _{2} 2 \pi n e+\frac{1}{2} \log _{2}\left(p_{1} p_{2} p_{3}\right),
$$

which coincides with the expression (10) in the limit $L=\infty$ for the case of spin $\sigma=1$. A more detailed analysis, analogous to the one done in [5], restores the finite-size dependence on $L$ of the von Neumann entropy as

$$
S(n) \approx \log _{2}\left(2 \pi e \frac{n(L-n)}{L}\right)+\frac{1}{2} \log _{2}\left(p_{1} p_{2} p_{3}\right) \text { for } \sigma=1 .
$$

Working out the same procedure for an arbitrary spin $\sigma$ we obtain Eq. (10).
[1] A. Osterloh, L. Amico, G. Falci, and R. Fazio, Nature (London) 416, 608 (2002).

[2] G. Vidal, J. I. Latorre, E. Rico, and A. Kitaev, Phys. Rev. Lett. 90, 227902 (2003).

[3] J. I. Latorre, E. Rico, and G. Vidal, e-print quant-ph/0304098, Quantum Inf. Comput. 4, 048 (2004).

[4] V. E. Korepin, Phys. Rev. Lett. 92, 096402 (2004).

[5] V. Popkov and M. Salerno, Phys. Rev. A 71, 012301 (2005).
[6] G. Albertini, V. E. Korepin, and A. Schadschneider, J. Phys. A 28, L303 (1995).

[7] B. Tóth, J. Stat. Phys. 61, 749 (1990).

[8] O. Penrose, J. Stat. Phys. 63, 761 (1991).

[9] M. Salerno, Phys. Rev. E 50, 4528 (1994).

[10] G. M. Schütz, "Exactly solvable models for many-body systems far from equilibrium," Phase Transitions and Critical Phenomena, edited by C. Domb and J. L. Lebowitz (Academic, London, 2000), Vol. 19. 
[11] H. J. Lipkin, N. Meshkov, and A. J. Glick, Nucl. Phys. 62, 188 (1965).

[12] P. Calabrese and J. Cardy, J. Stat. Mech.: Theory Exp. P06002 (2004).
[13] B. Sutherland, Phys. Rev. B 12, 3795 (1975).

[14] J. S. Pratt, Phys. Rev. Lett. 93, 237205 (2004).

[15] M. T. Batchelor and M. Maslen, J. Phys. A 32, L377 (1999).

[16] G. Schütz and S. Sandow, Phys. Rev. E 49, 2726 (1994). 